TITLE:

\title{
Fine-tuning in gauge mediated supersymmetry breaking models and induced top Yukawa coupling
}

AUTHOR(S):

Kobayashi, T; Terao, H; Tsuchiya, A

\section{CITATION:}

Kobayashi, T ...[et al]. Fine-tuning in gauge mediated supersymmetry breaking models and induced top Yukawa coupling. PHYSICAL REVIEW D 2006, 74(1): 015002.

\section{ISSUE DATE:}

2006-07

URL:

http://hdl.handle.net/2433/50539

RIGHT:

Copyright 2006 American Physical Society 
PHYSICAL REVIEW D 74, 015002 (2006)

\title{
Fine-tuning in gauge mediated supersymmetry breaking models and induced top Yukawa coupling
}

\author{
Tatsuo Kobayashi* \\ Department of Physics, Kyoto University, Kyoto 606-8502, Japan \\ Haruhiko Terao ${ }^{\dagger}$ and Akito Tsuchiya ${ }^{\ddagger}$ \\ Institute for Theoretical Physics, Kanazawa University, Kanazawa 920-1192, Japan
}

(Received 11 April 2006; published 5 July 2006)

\begin{abstract}
It is shown that fine-tuning of the Higgs parameters stronger than a few percent is required at best in the models with gauge mediated supersymmetry breaking. With the aim of solving this problem, we consider a new type of models in which the top Yukawa coupling is induced at $\mathrm{TeV}$ scale through mass mixing with unknown matter fields. Then it is found that the fine-tuning problem can be eliminated essentially. We discuss some phenomenological features of this model and also consider the extension to the next-tominimal models.
\end{abstract}

DOI: 10.1103/PhysRevD.74.015002

PACS numbers: $12.60 . J v, 14.65 . \mathrm{Ha}, 14.80 . \mathrm{Bn}$

\section{INTRODUCTION}

The supersymmetric extension of the standard model $(\mathrm{SM})$ is the most promising candidate for new physics beyond the $\mathrm{TeV}$ scale. Among many attractive features, the gauge coupling unification in the supersymmetric models is remarkable. However the fact that the Higgs boson besides any superparticle has not been discovered by the LEP2 experiment casts the so-called "little hierarchy problem" $[1,2]$ on this direction.

The lower experimental bound of the lightest Higgs boson mass, which is $114 \mathrm{GeV}$ [3], indicates that the mass should be lifted up by a large radiative correction of the top quark loop [4,5]. For the minimal supersymmetric standard model (MSSM), the mass of top squark (stop), $m_{\tilde{t}}$, is required to be much larger than the weak scale in general. The Higgs mass bound, which is also rather sensitive to the trilinear coupling of top squarks and Higgs boson, $A_{t}$, leads

$$
\begin{aligned}
& m_{\tilde{t}} \geq 500 \mathrm{GeV} \text { for }\left|A_{t}\right| \sim\left|m_{\tilde{t}}\right|, \\
& m_{\tilde{t}} \geq 1 \mathrm{TeV} \text { for }\left|A_{t}\right| \ll\left|m_{\tilde{t}}\right|,
\end{aligned}
$$

for a large value of $\tan \beta$. Moreover this mass bound increases rapidly, as $\tan \beta$ decreases. This "hierarchy" between the weak scale and the supersymmetry breaking mass scale turns out to bring about a severe fine-tuning problem as follows.

The quartic couplings of the Higgs fields are related with the gauge couplings in the MSSM. Therefore the scale of electroweak symmetry breaking (EWSB) is determined solely by the mass parameters of the Higgs bosons. For a moderate value of $\tan \beta$, such relation is simply given by

\footnotetext{
*Electronic address: kobayash@gauge.scphys.kyoto-u.ac.jp

Electronic address: terao@hep.s.kanazawa-u.ac.jp

Electronic address: tsucchi-@hep.s.kanazawa-u.ac.jp
}

$$
\frac{M_{Z}^{2}}{2}=-\mu^{2}-m_{H_{u}}^{2},
$$

where $\mu$ denotes the supersymmetric mass of the Higgs multiplets (or the Higgsino mass) and $m_{H_{u}}^{2}$ denotes the soft supersymmetry breaking mass (soft scalar mass) of the uptype Higgs boson $H_{u}$. This relation indicates that both $\mu$ and $\left|m_{H_{u}}\right|$ should be of the order of the weak scale, otherwise very accidental cancellation is required between them.

However, the soft scalar mass $m_{H_{u}}^{2}$ receives a fairly large radiative correction due to the very heavy stop mass and the large top Yukawa coupling $y_{t}$. Supersymmetry protects scalar masses from quadratic corrections, but not from logarithmic corrections. The correction to $m_{H_{u}}^{2}$ at the one-loop level is given approximately by

$$
\delta m_{H_{u}}^{2} \sim-\frac{3}{4 \pi^{2}} y_{t}^{2} m_{\tilde{t}}^{2} \ln \frac{\Lambda_{\mathrm{MSSM}}}{m_{\tilde{t}}},
$$

where $\Lambda_{\text {MSSM }}$ denotes the upmost scale of the MSSM. If we take this scale to be the scale of the grand unified theory (GUT), $\Lambda_{\text {GUT }} \sim 2 \times 10^{16} \mathrm{GeV}$, then this correction is estimated as $\left|\delta m_{H_{u}}^{2}\right| \geq(700 \mathrm{GeV})^{2}$ for $m_{\tilde{t}} \geq 500 \mathrm{GeV}$. Indeed this negative correction to the Higgs soft mass induces the radiative EWSB [6], which is one of the beautiful features of the supersymmetric models. Now, however, it is found that the size of the correction is too large. The realistic EWSB does not occur in the MSSM without delicate fine-tuning, since the Higgs mass parameters $\mu$ and $m_{H_{u}}$ are not related with each other in origin. Therefore, we may expect that the correction to the Higgs soft mass is suppressed for some reason.

Somewhat small sizes of the mass parameters $\mu$ and $\left|m_{H_{u}}\right|$ are favorable from the point of view of the dark matter as well. In the supersymmetric theories, the lightest superparticle (LSP), which is stable due to the $R$-parity symmetry, is supposed to be a good candidate for the dark 
matter. However, if the Higgsino mass $\mu$ is larger than the bino mass, then the dark matter relic density far exceeds the observed amount. On the other hand, when $\mu$ is comparable with the bino mass, the LSP is composed of a binoHiggsino mixture, which can lead to a suitable amount of the relic density [7].

Thus it would be worthwhile to ask what kinds of models instead of the MSSM offer us more natural frameworks. The gauge mediated supersymmetry breaking (GMSB) models [8] may be thought to be a good candidate, since the uppermost scale $\Lambda_{\text {MSSM }}$ can be lowered to about $50 \mathrm{TeV}$. Then the large logarithmic factor in (3) is reduced to $4 \sim 5$. However, it has been known that the finetuning in the GMSB models is not improved, rather it becomes worse because of the following reasons.

In the GMSB framework, the messenger fields, which acquire the soft scalar masses through interaction with the dynamical supersymmetry breaking (DSB) sector, decouples at the messenger scale $\Lambda_{\text {mess }}$. Then the ratio among the various soft supersymmetry breaking parameters induced at $\Lambda_{\text {mess }}$ is given uniquely by the SM gauge couplings. In the case of the low scale GMSB with e.g. $\Lambda_{\text {mess }} \sim 50 \mathrm{TeV}$, the ratio of soft masses of the stop $m_{\tilde{t}}$ and of the righthanded selectron $m_{\tilde{e}}$ may be evaluated roughly as

$$
\frac{m_{\tilde{t}}^{2}}{m_{\tilde{e}}^{2}} \simeq \frac{(4 / 3) g_{3}^{4}}{(3 / 5) g_{1}^{4}} \simeq(7 \sim 8)^{2} .
$$

Meanwhile, the slepton mass is bounded experimentally as $m_{\tilde{e}} \geq 100 \mathrm{GeV}$. Therefore $m_{\tilde{t}}$ appears to be quite large, $m_{\tilde{t}} \geq 700 \mathrm{GeV}$, in the low scale GM. Such a large stop mass makes the correction to the Higgs soft mass even bigger in spite of the smaller logarithmic factor $[9,10]$.

It should be also noted that $A$-parameters are not induced or very suppressed at the messenger scale, except for special cases with vector messengers at the GUT scale. Therefore the trilinear coupling $A_{t}$ remains small up to low energy in the low scale GMSB. Then the Higgs mass bound imposes a very severe constraint on the stop mass $m_{\tilde{t}}$.

It has been also known that the GMSB has a difficulty to lead the proper size of $B$-parameter as well as the $\mu$-parameter $[8,11]$. The easiest solution escaping this $\mu$-problem would be to consider the next-to-MSSM (NMSSM) type extension [12]. There we introduce a SM gauge singlet $N$ and consider the superpotential

$$
W=\lambda_{H} N H_{u} H_{d}+\cdots \text {. }
$$

The $\mu$-parameter is given by the nonvanishing vacuum expectation value (vev) of $N$ as $\mu=\lambda_{H}\langle N\rangle$. Then the $B$-parameter in the MSSM is just given by the trilinear coupling $A_{H}$ of the scalar fields,

$$
V=-\lambda_{H} A_{H} N H_{u} H_{d}+\cdots
$$

The problem is that $\tan \beta$ is linked to the soft scalar mass of the singlet $N$ and cannot be chosen freely contrary to the MSSM. In practice, it will be seen later that $\tan \beta$ is restricted to be small in the realistic models [10]. Then the experimental Higgs mass bound leads to a very strong constraint on the soft scalar mass $m_{\tilde{t}}$ in general.

So far various approaches have been proposed aiming to solve this "little hierarchy problem" [13-24]. Many of them push up the Higgs mass bound by introducing other new interactions and assume a fairly small $m_{\tilde{t}}$ to suppress the correction to the Higgs mass. The GMSB seems inappropriate in these approaches, since the mass bound for the selectron leads to a large stop mass anyway. Apart from the GMSB, it is also a way to consider special setup generating the soft breaking parameters at some high energy scale such that $\left|m_{H_{u}}\right|$ appears to be small enough at the weak scale, as e.g. the focus point solution [25], though the ground leading to the special supersymmetry breaking parameters assumed there is missing. Another interesting scenario is the mixed modulus-anomaly mediation, the socalled mirage mediation. In a certain class of such models the logarithmic correction (3) is canceled by the anomaly mediation effect, and that leads to the favorable spectrum that stop is heavy and the soft scalar mass $\left|m_{H_{u}}\right|$ is small, while $\left|A_{t}\right|$ is large [21]. The recently proposed setup with a negative $m_{\tilde{t}}^{2}$ at the GUT scale [22] would be also an interesting possibility in this point of view.

Another type of approach is to protect the Higgs mass from the large radiative corrections. The well-known examples of this kind are the (supersymmetric) little Higgs models [17], where the Higgs scalar is the pseudo-NG boson and the logarithmic divergence is suppressed by two-loop factors. Two of the authors have also proposed the models, in which the Higgs mass correction is made even finite, i.e. cutoff independent, by use of the superconformal dynamics [18,19]. In these approaches a large $m_{\tilde{t}}$ is also admitted and, therefore, the GMSB can be incorporated in the models.

In this paper we would like to consider this problem in relation to the origin of prominently large top quark mass. It is obvious that the correction to the Higgs mass is not so enhanced, if the top Yukawa coupling $y_{t}$ is small above $\mathrm{TeV}$ scale. Here we consider the models in which the top Yukawa coupling is induced effectively at $\mathrm{TeV}$ scale through mixing between top quarks and extra vectorlike particles [19]. In order to get the top Yukawa coupling large enough, the Higgs field is assumed to be coupled with the extra particles rather strongly.

Furthermore we consider the "partial GMSB" to protect the Higgs soft mass from a large loop correction of the extra particles. The gauge group of the model is assumed to be a direct product of the SM gauge group $G_{\mathrm{SM}}$ and the extra gauge group $G_{\text {ex }}$. We suppose also that the extra vectorlike fields are neutral to the color $S U(3)$, while the gauge messengers are neutral to $G_{\mathrm{ex}}$. Then all other scalar masses than the squark masses; the soft scalar masses of the Higgs, the sleptons and the extra particles, become small automatically. The gluino is also heavy as in the 
ordinary GMSB. The point is that the squark masses do not enhance the Higgs mass, since the top Yukawa coupling is assumed to be small above $\mathrm{TeV}$ scale. It may be also said that the large logarithmic correction to the Higgs mass by the stop loop is cutoff effectively at $\mathrm{TeV}$ scale. Consequently, our model realizes the fairly small Higgs mass parameters at the weak scale in spite of the large stop masses, and, therefore, is released from the fine-tuning problem.

This paper is organized as follows. In Sec. II we examine the degree of fine-tuning in the GMSB models with taking account of the Higgs mass bound by LEP2. It will be seen that the delicate fine-tuning within a few percent level is required at the very best. In Sec. III we present an explicit model with the induced top Yukawa coupling and discuss some phenomenological features. Then we quantify the degree of fine-tuning required in the new model explicitly in Sec. IV. In Sec. V we consider the extension to the NMSSM type in order to solve the $\mu$-problem. The last section is devoted to the conclusion.

\section{FINE-TUNING IN THE GMSB MODELS}

We consider the minimal GMSB model with vectorlike chiral messenger fields $(q, l)$ and $(\bar{q}, \bar{l})$. The fields $q$ and $l$ transform as $(\mathbf{3}, \mathbf{1},-\mathbf{1} / \mathbf{3})$ and $(\mathbf{1}, \mathbf{2}, \mathbf{1} / \mathbf{2})$ under the SM gauge group $G_{\mathrm{SM}}=S U(3)_{c} \times S U(2)_{W} \times U(1)_{Y}$, respectively, and $(\bar{q}, \bar{l})$ carry their conjugate representations. These fields do not have any direct interactions with the MSSM sector. Since the fields $q$ and $l$ are combined into a 5 representation of the $S U(5)$ group, the gauge coupling unification is maintained.

In the minimal GMSB model, the messengers couple with the DSB sector through the superpotential given by

$$
W_{\mathrm{GMSB}}=\kappa_{q} S q \bar{q}+\kappa_{l} S l \bar{l},
$$

where $S$ is a SM gauge singlet. The dynamics of the DSB sector is supposed to generate nonvanishing vevs for $S$ and the $F$-component $F_{S}$ at the same time. These messenger decouples from the MSSM sector at the messenger scale $\Lambda_{\text {mess }} \sim \kappa\langle S\rangle$.

The $F$-component vev induces soft supersymmetry breaking parameters in the MSSM sector as finite loop corrections by the messenger fields. The formulas for the induced parameters are given at the leading order by

$$
\begin{gathered}
M_{a}=\frac{\alpha_{a}}{4 \pi} B_{S} \quad(a=1,2,3), \\
m_{\tilde{f}_{i}}^{2}=2\left[C_{3 i}\left(\frac{\alpha_{3}}{4 \pi}\right)^{2}+C_{2 i}\left(\frac{\alpha_{2}}{4 \pi}\right)^{2}+\frac{3}{5} Y_{i}^{2}\left(\frac{\alpha_{1}}{4 \pi}\right)^{2}\right] B_{S}^{2}, \\
A_{i}=0,
\end{gathered}
$$

where $B_{S}=\left\langle F_{S}\right\rangle /\langle S\rangle$, and $C_{2}, C_{3}, Y$ denote the quadratic Casimirs of the representation for $S U(2)_{W}$ and $S U(3)_{c}$ and the hypercharge for $U(1)_{Y}$, respectively. It is a general property that the induced $A$-parameter is very small at the messenger scale in the chiral messenger GMSB. Although some special models of GMSB by vector messengers may have sizable $A$-parameters [26], we do not consider such cases in this paper.

Here we note that all the soft scalar masses are fixed up to the overall scale of the supersymmetry breaking parameter $B_{S}$, which is also bounded as discussed below. Therefore we can tune only the $\mu$ parameter in the GMSB to satisfy the minimization condition for the Higgs potential,

$$
\frac{M_{Z}^{2}}{2}=-\mu^{2}+\frac{m_{H_{d}}^{2}-m_{H_{u}}^{2} \tan ^{2} \beta}{\tan ^{2} \beta-1} .
$$

The degree of fine-tuning may be defined by

$$
\Delta_{\mu^{2}}\left(M_{Z}^{2}\right)=\left|\frac{\mu^{2}}{M_{Z}^{2}} \frac{\partial M_{Z}^{2}}{\partial \mu^{2}}\right|=2 \frac{\mu^{2}}{M_{Z}^{2}}
$$

These soft mass parameters are subject to the renormalization group (RG) of the MSSM below the messenger scale. The soft mass of the right-handed selectron $m_{\tilde{e}}$ at the weak scale is given roughly by

$$
\begin{aligned}
m_{\tilde{e}}^{2} \sim & \frac{6}{5}\left(\frac{\alpha_{1}}{4 \pi}\right)^{2} B_{S}^{2}+\frac{2}{11}\left[\left(\frac{\alpha_{1}\left(\Lambda_{\text {mess }}\right)}{\alpha_{1}\left(M_{Z}\right)}\right)^{2}-1\right] M_{1}^{2}\left(M_{Z}\right) \\
& -M_{Z}^{2} \sin ^{2} \theta_{W} \cos 2 \beta,
\end{aligned}
$$

where $\theta_{W}$ denotes the Weinberg angle and $\sin ^{2} \theta_{W} \simeq 0.232$. The experimental lower bound $m_{\tilde{e}} \geq 100 \mathrm{GeV}$ constrains the supersymmetry breaking parameter $B_{S}$ to be more than roughly $50 \mathrm{TeV}$. Therefore it would be reasonable to take the messenger scale $\Lambda_{\text {mess }}>50 \mathrm{TeV}$ for the typical low scale GMSB, provided the DSB sector induces $\left\langle F_{S}\right\rangle \leq\langle S\rangle^{2}$ [27].

In such a low scale GMSB case, the logarithmic factor in the one-loop correction given by (3) is somewhat reduced, $\log \left(\Lambda_{\text {mess }} / m_{\tilde{t}}\right) \sim 5$. However, the soft scalar mass $m_{\tilde{t}}$ is quite large instead. In practice it is found by solving the one-loop RG equations that $m_{\tilde{t}} / m_{\tilde{e}} \sim 7$ at the weak scale. Therefore $m_{\tilde{t}}>700 \mathrm{GeV}$ is required from the mass bound to (13) for large $\tan \beta$. Then the soft mass of the Higgs turns out to be $m_{H_{u}}^{2}<-(300 \mathrm{GeV})^{2}$ at the weak scale by the one-loop RG analysis. Then this result shows that about $5 \%$ fine-tuning for the $\mu^{2}$ parameter is required at the best $[9,10]$. For a small value of $\tan \beta$, the required stop mass becomes slightly bigger, therefore the fine-tuning is worse.

In practice, however, the experimental Higgs mass bound, $m_{h^{0}}>114.4 \mathrm{GeV}$, is found to put a much stronger constraint than the selectron mass bound does. Within the two-loop approximation, the analytic expression for the mass bound has been given by [5] 
TATSUO KOBAYASHI et al.

$$
\begin{aligned}
m_{h^{0}}^{2} \leq & M_{Z}^{2} \cos ^{2} 2 \beta\left(1-\frac{3 m_{t}^{2}}{8 \pi^{2} v^{2}} t\right)+\frac{3 m_{t}^{4}}{4 \pi^{2} v^{2}}\left[\frac{1}{2} \tilde{X}_{t}+t\right. \\
& \left.+\frac{1}{16 \pi^{2}}\left(\frac{3 m_{t}^{2}}{2 v^{2}}-32 \pi \alpha_{3}\right)\left(\tilde{X}_{t} t+t^{2}\right)\right]
\end{aligned}
$$

where $t=\log m_{\tilde{t}}^{2} / m_{t}^{2}$ defined with the average stop mass $m_{\tilde{t}}^{2}=\sqrt{m_{\tilde{Q}_{3}}^{2} m_{\tilde{u}_{3}}^{2}}\left(m_{\tilde{Q}_{3}}^{2}\right.$ and $m_{\tilde{u}_{3}}^{2}$ denote the soft scalar masses for the left-handed stop-sbottom doublet and the right-handed stop, respectively), $v=173.7 \mathrm{GeV}$ is the vev of Higgs and $m_{t}$ is the on-shell running mass computed from the physical top mass $M_{t}=172.7 \mathrm{GeV}$, i.e. $m_{t}=$ $164.5 \mathrm{GeV}$. The factor $\tilde{X}_{t}$ stands for the mixing between the left- and right-handed stops and is given explicitly by

$$
\tilde{X}_{t}=\frac{2 \tilde{A}_{t}^{2}}{m_{\tilde{t}}^{2}}\left(1-\frac{\tilde{A}_{t}^{2}}{12 m_{\tilde{t}}^{2}}\right),
$$

where $\tilde{A}_{t}=A_{t}-\mu \cot \beta$. In this analytic expression, the maximal value of mixing is $\tilde{X}_{t}=6$, which is realized for $\tilde{A}_{t} / m_{\tilde{t}}=\sqrt{6}$. The constraint on $m_{\tilde{t}}$ imposed by the Higgs mass bound is rather sensitive to the stop mixing and $m_{\tilde{t}}$ is required to be more than $1 \mathrm{TeV}$ for a small $A_{t}$.

In the GMSB, in which $A_{t}=0$ at the messenger scale $\Lambda_{\text {mess }}$, the value of $A_{t}$ at the weak scale is given approximately by

$$
A_{t} \sim-\frac{8}{3 \pi} \alpha_{3} M_{3} \log \left(\frac{\Lambda_{\text {mess }}}{m_{\tilde{t}}}\right) .
$$

Therefore, $\left|A_{t}\right|$ is rather small for the low scale GMSB and $\left|A_{t}\right| \leq m_{\tilde{t}}$ for any messenger scale $\Lambda_{\text {mess }}<\Lambda_{\mathrm{GUT}}$. This is a crucial feature of the GMSB models for the fine-tuning problem.

In Figs. 1 and 2, the minimum degrees of fine-tuning required in the MSSM are shown in the cases with $\tan \beta=$

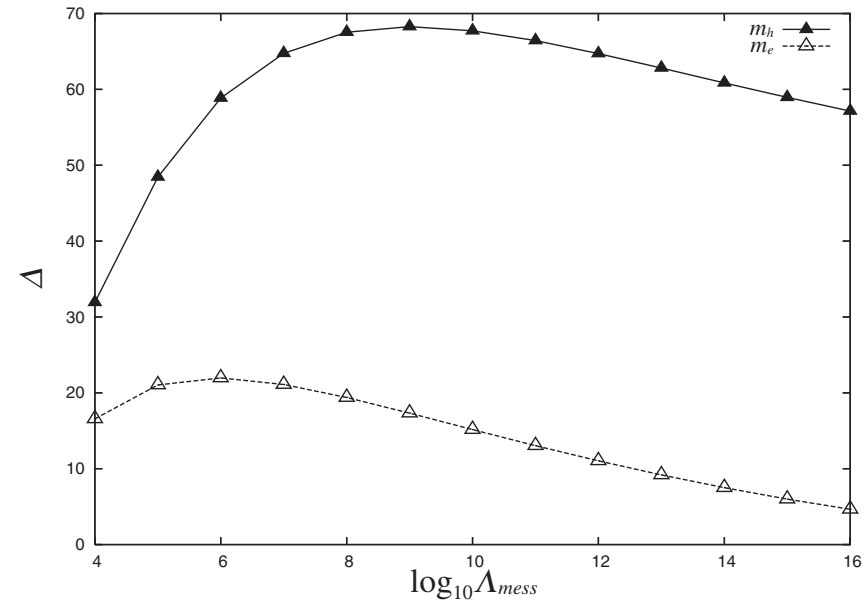

FIG. 1. The minimum degrees of fine-tuning required in the MSSM with $\tan \beta=10$ are shown with respect to various messenger scales $\Lambda_{\text {mess }}$ of the GMSB. The upper line is given by the Higgs mass bound, while the lower line is given by the selectron mass bound.
PHYSICAL REVIEW D 74, 015002 (2006)

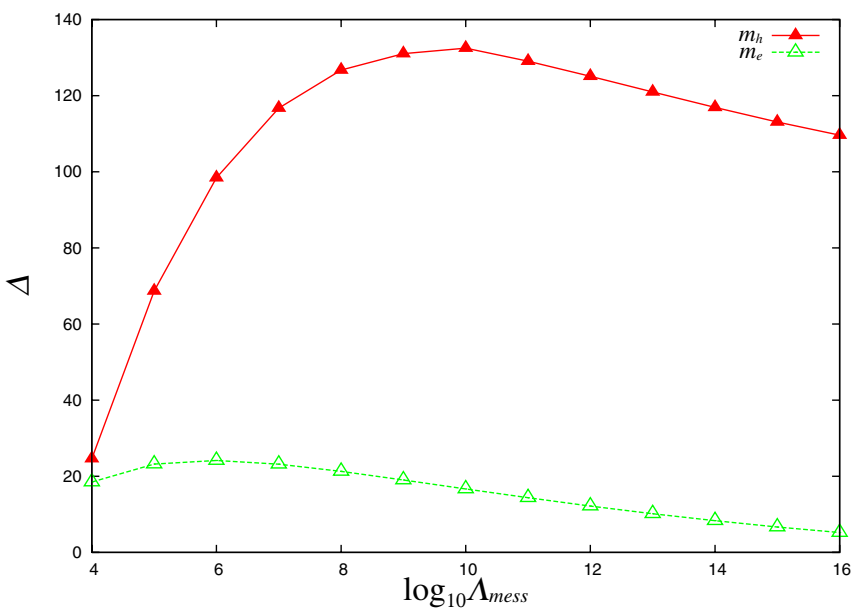

FIG. 2 (color online). The minimum degrees of fine-tuning required in the MSSM with $\tan \beta=5$ are shown with respect to various messenger scales $\Lambda_{\text {mess }}$ of the GMSB. The conditions for the lines are the same as in Fig. 1.

10 and 5, respectively. The messenger scale $\Lambda_{\text {mess }}$ is varied up to the GUT scale. It is seen that the Higgs mass bound puts a far stronger constraint on the fine-tuning than the selectron mass bound. Both the constraints become milder as the messenger scale goes higher. However the finetuning stronger than $2 \%$ is found to be required at the very best. For the case with $\tan \beta<5$, the fine-tuning is more severe than the $1 \%$ level except for the case with a very low scale messenger scale. The dependence of $\Delta_{\mu^{2}}$ on the $\tan \beta$ parameter in the case of a high messenger scale is shown in Fig. 3. It is seen that the bound by the lightest Higgs boson mass becomes extremely severe for a small $\tan \beta<5$ [28].

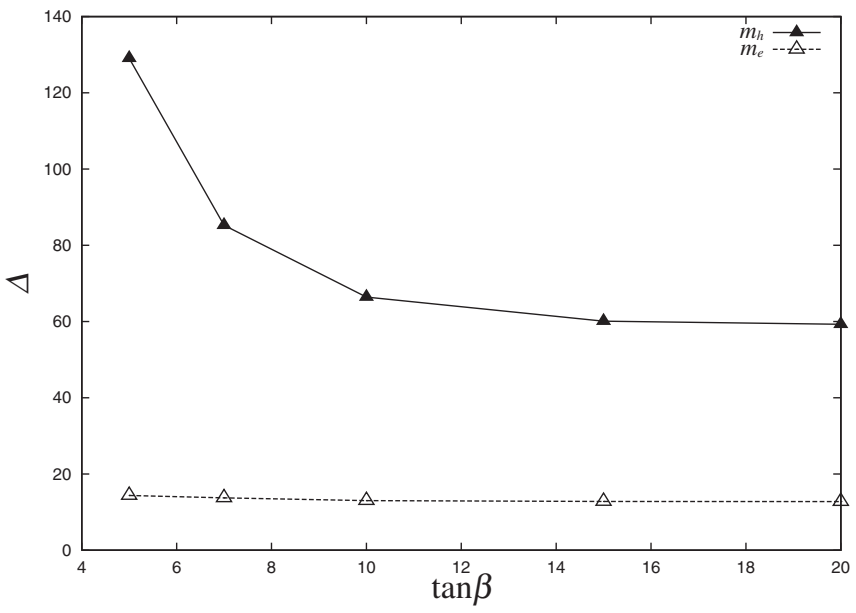

FIG. 3. The minimum degrees of fine-tuning required in the MSSM with the messenger scale $\Lambda_{\text {mess }}=10^{16} \mathrm{GeV}$ are shown for various values of $\tan \beta$. The conditions for the lines are the same as in Fig. 1. 


\section{EXTRA $S U(3)$ MODEL WITH THE INDUCED TOP YUKAWA COUPLING}

\section{A. The extra $S U(3)$ model}

In this section, we consider an explicit model avoiding this severe fine-tuning problem within the GMSB framework. The new model is based on the ideas of the "induced top Yukawa coupling" and the "partial gauge mediation." Although the induced soft scalar mass of the Higgs field $H_{u}$ by the gauge messengers is not large, the large soft mass transmitted through the stop loop causes the problematic hierarchy. If the top Yukawa coupling is as small as other Yukawa couplings above $\mathrm{TeV}$ scale, then this transmission can be effectively switched off. Naturally all other scalar fields than squarks in the MSSM, including the Higgs scalars, carry relatively small soft scalar masses.

Meanwhile it is an interesting puzzle of the flavor physics why only the top quark is so heavy. Here we take the picture that the large top Yukawa coupling is induced at $\mathrm{TeV}$ scale dynamically. There must be the additional sector coupled with the Higgs field so strongly as to generate the large top Yukawa coupling. However, if the supersymmetry breaking is not mediated to the new sector, then the Higgs field does not receive sizable corrections in spite of the large coupling to the new sector. This is the reason why we apply the gauge mediation acting partially.

First we assume that the SM gauge group is extended to $G=S U(3)_{\mathrm{ex}} \otimes S U(3)_{C}^{\prime} \otimes S U(2)_{W} \otimes U(1)_{Y} \quad$ and that $S U(3)_{\mathrm{ex}} \otimes S U(3)_{C}^{\prime}$ is spontaneously broken to the color $S U(3)_{C}$ diagonally at a few $\mathrm{TeV}$ scale. We introduce also vectorlike matter fields charged under $G$ as

\begin{tabular}{|c|ccccc|}
\hline & $S U(3)_{e x}$ & $S U(3)_{C}^{\prime}$ & $S U(2)_{W}$ & $U(1)_{Y}$ & $R$ \\
\hline$\Phi$ & $\mathbf{3}$ & $\mathbf{1}$ & $\mathbf{2}$ & $1 / 6$ & - \\
$\bar{\Phi}$ & $\mathbf{3}^{*}$ & $\mathbf{1}$ & $\mathbf{2}$ & $-1 / 6$ & - \\
$\phi$ & $\mathbf{3}$ & $\mathbf{1}$ & $\mathbf{1}$ & $2 / 3$ & - \\
$\bar{\phi}$ & $\mathbf{3}^{*}$ & $\mathbf{1}$ & $\mathbf{1}$ & $-2 / 3$ & - \\
$\Omega$ & $\mathbf{3}$ & $\mathbf{3}^{*}$ & $\mathbf{1}$ & 0 & + \\
$\bar{\Omega}$ & $\mathbf{3}^{*}$ & $\mathbf{3}$ & $\mathbf{1}$ & 0 & + \\
\hline
\end{tabular}

where $R$ denotes the $R$-parity. The superpotential of the model is given by

$$
\begin{array}{r}
W=W_{\mathrm{GMSB}}+W_{\mathrm{MSSM}}+W_{\text {yukawa }}+W_{\text {mass }}, \\
W_{\mathrm{MSSM}}=y_{t} Q_{3} \bar{u}_{3} H_{u}+\mu H_{u} H_{d}+\cdots, \\
W_{\text {yukawa }}=y_{\Phi} \Phi \bar{\phi} H_{u}+x Q_{3} \Omega \bar{\Phi}+\bar{x} \phi \bar{\Omega} \bar{u}_{3}, \\
W_{\text {mass }}=M_{\Phi} \Phi \bar{\Phi}+M_{\phi} \phi \bar{\phi}+M_{\Omega} \Omega \bar{\Omega} .
\end{array}
$$

It is noted that the quarks not only of the third generation but also of an arbitrary combination of the generations may couple to the extra fields in $W_{\text {yukawa }}$. Then such a combination of quarks turns out to be heavy and is regarded as the top quark in the end. We do not take account of the new couplings with $H_{d}$ either. If the induced bottom Yukawa coupling is small for some reason, then it will be also viable.

In the above superpotential, the explicit mass terms for the extra fields in $W_{\text {mass }}$ are introduced by hand. To be explicit we simply set $M_{\Phi}=M_{\phi}=M_{\Omega}=5 \mathrm{TeV}$ hereafter, though somewhat smaller mass parameters are also allowed by the EW precision experiments as is seen later on. Then below $5 \mathrm{TeV}$, the extra sector decouples from the MSSM sector. It is, indeed, a problem that these mass operators can couple with the singlet $S$ with nonvanishing $F$-component as well. Actually this is the same problem as the $\mu$-problem in the GMSB $[8,11]$ and these couplings should be forbidden e.g. by the discrete $U(1)_{P Q}$ symmetry. We shall take care of these problems altogether in the extension to the next-to-MSSM type discussed in Sec. V. There it will be also shown that the hierarchy between $\mu$ and the decoupling mass scale can be generated naturally by the strong gauge interaction of $S U(3)_{\mathrm{ex}}[19]$.

We assume the top Yukawa coupling $y_{t}$ in the superpotential given by (19) is as small as, e.g., the bottom Yukawa coupling. The explicit size of $y_{t}$ is irrelevant in our discussions. Rather, the large top Yukawa coupling is induced from $y_{\Phi}$ through the mass mixing between the top quarks and the extra fields at the spontaneous symmetry breaking (SSB). Hence $y_{\Phi}$ should be larger than 1 at the decoupling scale. Indeed the extra sector is the same as the $S U(3)$ QCD with 6 flavors, which belong to the so-called conformal window [29]. Therefore the Yukawa coupling as well as the extra gauge coupling satisfy an infrared (IR) attractive fixed point $[18,19]$. In this case, the fixed point lies in the strongly coupled region. Thus such a large Yukawa coupling is realized naturally. In the next section we shall discuss this in more detail.

The SSB of $S U(3)_{\mathrm{ex}} \otimes S U(3)_{C}^{\prime}$ to the color $S U(3)_{C}$ is brought about by nonvanishing vevs of $\Omega$ and $\bar{\Omega}$. If the extra gauge coupling grows up around the decoupling scale, then some strong dynamics may generate these vevs of the $\mathrm{TeV}$ order. Here we do not study on explicit dynamics leading to the vevs, but rather assume

$$
\left\langle\Omega_{a}^{A}\right\rangle=\omega \delta_{a}^{A}, \quad\left\langle\bar{\Omega}_{A}^{a}\right\rangle=\bar{\omega} \delta_{A}^{a},
$$

where $A$ and $a(=1,2,3)$ denote indices of the fundamental representations of $S U(3)_{\mathrm{ex}}$ and $S U(3)_{C}^{\prime}$ respectively. The vevs $\omega$ and $\bar{\omega}$ are also given to be several TeV.

After this SSB, the mass terms in the superpotential (21) are modified to

$$
M_{\Phi}\left(\Phi+\frac{x \omega}{M_{\Phi}} Q_{3}\right) \bar{\Phi}+M_{\phi} \phi\left(\bar{\phi}+\frac{\bar{x} \bar{\omega}}{M_{\phi}} \bar{u}_{3}\right) .
$$

These mass terms induce mixing between $S U(2)_{W}$ doublets, $Q_{3}$ and $\Phi^{\prime}$, and also singlets, $\bar{u}_{3}$ and $\bar{\phi}$. The mass 
eigenmodes $\left(Q_{3}^{\prime}, \Phi^{\prime}\right)$ and $\left(\bar{u}_{3}^{\prime}, \bar{\phi}^{\prime}\right)$ are given as

$$
\begin{aligned}
\left(\begin{array}{l}
Q_{3}^{\prime} \\
\Phi^{\prime}
\end{array}\right)=\left(\begin{array}{cc}
\cos \theta_{L} & -\sin \theta_{L} \\
\sin \theta_{L} & \cos \theta_{L}
\end{array}\right)\left(\begin{array}{c}
Q_{3} \\
\Phi
\end{array}\right), \\
\left(\begin{array}{c}
\bar{u}_{3}^{\prime} \\
\bar{\phi}^{\prime}
\end{array}\right)=\left(\begin{array}{cc}
\cos \theta_{R} & -\sin \theta_{R} \\
\sin \theta_{R} & \cos \theta_{R}
\end{array}\right)\left(\begin{array}{c}
\bar{u}_{3} \\
\bar{\phi}
\end{array}\right),
\end{aligned}
$$

where $\tan \theta_{L}=x \omega / M_{\Phi}$ and $\tan \theta_{R}=\bar{x} \bar{\omega} / M_{\phi}$. These mixing angles are supposed to be of order one, since the vevs $\omega$ and $\bar{\omega}$ are assumed to be of the same order of the decoupling mass scale. The massless modes $Q_{3}^{\prime}$ and $\bar{u}_{3}^{\prime}$ are regarded to be the third generation quarks in the effective MSSM. The massive modes are given by $\left(\Phi^{\prime}, \bar{\Phi}\right)$ and of $\left(\phi, \bar{\phi}^{\prime}\right)$, whose masses are slightly modified to $M_{\Phi}^{\prime}=$ $M_{\Phi} / \cos \theta_{L}$ and $M_{\phi}^{\prime}=M_{\phi} / \cos \theta_{R}$.

After decoupling of the heavy particles, the theory is reduced to be the MSSM with the additional effective top Yukawa interaction,

$$
\begin{gathered}
W \sim W_{\mathrm{MSSM}}+y_{\Phi}\left(\cos \theta_{L} \Phi^{\prime}-\sin \theta_{L} Q_{3}^{\prime}\right)\left(\cos \theta_{R} \bar{\phi}^{\prime}\right. \\
\left.-\sin \theta_{R} \bar{u}_{3}^{\prime}\right) H_{u} \sim W_{\mathrm{MSSM}}+y_{t}^{\mathrm{eff}} Q_{3}^{\prime} \bar{u}_{3}^{\prime} H_{u} .
\end{gathered}
$$

Here the induced top Yukawa coupling $y_{t}^{\text {eff }}$ is given explicitly by

$$
y_{t}^{\mathrm{eff}}=y_{\Phi} \sin \theta_{L} \sin \theta_{R}
$$

This coupling can be so large as to lead the physical top quark mass $M_{t}=172.2 \mathrm{GeV}$, since $y_{\Phi}$ is rather large.

Next we consider the soft supersymmetry breaking parameters obtained in the effective theory. We apply the same supersymmetry breaking sector and the gauge messengers $(q, \bar{q})$ charged to the $S U(3)_{C}^{\prime}$, in place of the $S U(3)_{C}$, and $(l, \bar{l})$. Note that these messengers are neutral to the $S U(3)_{\mathrm{ex}}$. Therefore the gauginos of the $S U(3)_{\mathrm{ex}}$ sector remain just massless all the way down to the low energy scale. After the diagonal SSB, the gauge coupling of the color $S U(3)_{C}$ is given by $1 / g_{3}^{2}=1 / g_{\mathrm{ex}}^{2}+1 / g_{3}^{\prime 2}$. Since the coupling $g_{\text {ex }}$ is fairly large, we have $g_{3} \sim g_{3}^{\prime}$. Meanwhile the gaugino masses, $M_{3}$ for the $S U(3)_{C}$ and $M_{3}^{\prime}$ for the $S U(3)_{C}^{\prime}$, at the SSB are related as

$$
\frac{M_{3}}{g_{3}^{2}}=\frac{M_{\mathrm{ex}}}{g_{\mathrm{ex}}^{2}}+\frac{M_{3}^{\prime}}{g_{3}^{/ 2}},
$$

which leads to $M_{3} \sim M_{3}^{\prime}$. Therefore the gaugino mass spectra obtained at low energy is almost the same as the conventional GMSB predicts.

The induced soft scalar masses $m_{\Phi}^{2}$ and $m_{\phi}^{2}$ for the extra fields $\Phi$ and $\phi$ are small at the messenger scale in general because these fields are neutral to the $S U(3)_{C}^{\prime}$. Besides, these soft masses are free from enhancement by the gaugino corrections. As a result of this nature, the soft mass of the Higgs scalar $m_{H_{u}}^{2}$ is not enhanced either in spite of the large Yukawa coupling $y_{\Phi}$. If we could switch off the gauge couplings of $S U(2)_{W}$ and $U(1)_{Y}$, then $m_{H_{u}}^{2}$ as well as $m_{\Phi}^{2}$ and $m_{\phi}^{2}$ were all vanishing in this partial GMSB. Thus the Higgs scalar can be "sequestered" from the large supersymmetry breaking sources. In practice, the effects of the $S U(2)_{W}$ and $U(1)_{Y}$ gauge interactions are not negligible. We postpone the quantitative analysis of the Higgs mass and the degree of fine-tuning to Sec. IV.

\section{B. Electroweak precision test}

In the extra $S U(3)$ model, the top quarks are mixed with the heavy fermions with TeV scale masses. In general, such mixing effect induces sizable corrections to the EW theory, while the corrections are restricted rather strictly by the precision measurements at the LEP experiments. Here we evaluate such corrections expected in the extra $S U(3)$ model explicitly.

First we evaluate the corrections to the EW oblique parameters, $T$ and $S$. In the extra $S U(3)$ model presented above, only the up-type quarks couple with the extra matter fields. Thus the custodial $S U(2)$ symmetry is largely broken and, therefore, the correction to $T$-parameter $\Delta T$ can be sizable. Of course the corrections are more suppressed, as the masses of the heavy fields are made larger by their decoupling effect. However we may find the lower bound for the decoupling mass scale allowed by the EW precision test.

Since the loop effects by the scalar particles are small due to the large soft masses, we take only the fermionic fields into account. To be explicit, we write the fermionic components of the superfields as

$$
\begin{array}{ccc}
Q_{3 L}^{\prime} \sim\left(t_{L}, b_{L}\right), & \bar{u}_{3 R}^{\prime} \sim t_{R}, \quad \Phi_{L}^{\prime} \sim\left(\chi_{L 1}, \chi_{L 2}\right), \\
\phi_{R}^{\prime} \sim \chi_{R}, \quad \Phi_{R} \sim\left(\chi_{R 1}, \chi_{R 2}\right), & \phi_{L} \sim \chi_{L} .
\end{array}
$$

These fermions also obtain the additional masses through the Yukawa interactions given in (26). The Higgs scalar's vev, $\left\langle H_{u}\right\rangle=\left(v_{u}, 0\right)$, for the EWSB leads to the fermion mass terms given by

$$
\begin{aligned}
& \left(\bar{t}_{L}, \bar{\chi}_{L 1}, \bar{\chi}_{L}\right)\left(\begin{array}{ccc}
y_{\Phi} s_{L} s_{R} v_{u} & 0 & -y_{\Phi} s_{L} c_{R} v_{u} \\
-y_{\Phi} c_{L} s_{R} v_{u} & M_{\Phi}^{\prime} & y_{\Phi} c_{L} c_{R} v_{u} \\
0 & y_{\Phi} v_{u} & M_{\phi}^{\prime}
\end{array}\right) \\
& \quad \times\left(\begin{array}{c}
t_{R} \\
\chi_{R 1} \\
\chi_{R}
\end{array}\right)+M_{\Phi}^{\prime} \bar{\chi}_{L 2} \chi_{R 2},
\end{aligned}
$$

where $s_{L(R)}=\sin \theta_{L(R)}$ and $c_{L(R)}=\cos \theta_{L(R)}$. This mass matrix shows that the EWSB induces mixing between the weak doublets and singlets of the left-handed fermions, $\left(t_{L}, \chi_{L}\right)$, and also of the right-handed fermions, $\left(t_{R}, \chi_{R 1}\right)$.

The additional oblique corrections are generated through the weak doublet-singlet mixing given above. By calculating the one-loop diagrams with $M_{\Phi}^{\prime}=M_{\phi}^{\prime}=M^{\prime}$, we may evaluate $\Delta T$ as 
FINE-TUNING IN GAUGE MEDIATED SUPERSYMMETRY ...

PHYSICAL REVIEW D 74, 015002 (2006)

$$
\Delta T \sim \frac{3}{16 \pi^{2} \alpha_{W}} \log \frac{M^{\prime 2}}{m_{t}^{2}}\left(\frac{y_{\Phi} v_{u}}{M^{\prime}}\right)^{2} \sim O(1) \times\left(\frac{y_{\Phi} v_{u}}{M^{\prime}}\right)^{2},
$$

unless the Yukawa coupling $y_{\Phi}$ is extremely large. On the other hand, the experimental bound is given roughly by $\Delta T \leq 0.1$ [30]. Consequently, we find that a fairly low decoupling mass $M^{\prime}$ up to about $1 \mathrm{TeV}$ is not excluded. It is noted that the above oblique correction has the common or very similar structure with the top quark seesaw models [31] and some of the little Higgs models [32].

Incidentally, other constraints imposed by the precision experiments are not restrictive. The correction to $S$-parameter turns out to be relatively smaller than $\Delta T$ by the one-loop calculation. Another experimental bound discussed frequently is the excess of the $Z$-boson decay, $R_{b}=$ $\Gamma(Z \rightarrow b \bar{b}) / \Gamma(Z \rightarrow$ hadrons $)$. However, as long as there is no (or a very small) mixing mass for the bottom quarks in the present model, the excess is also found to be much less than the experimental bound $\left(\delta R_{b}<1 \%\right)$. These features are also common with the top quark seesaw models [31].

\section{Sparticle spectra}

The extra fields do not have direct couplings with the MSSM fields except for the Higgs field $H_{u}$. The mass spectrum of these superparticles is determined in the GMSB, once the messenger scale $\Lambda_{\text {mess }}$ is fixed. Meanwhile, the masses of the Higgs and the Higgsino are very dependent on the running behavior of the Yukawa coupling $y_{\Phi}$, which will be discussed in the next section.

However, the presence of the extra fields alter the running behavior of the gauge couplings $g_{3}^{\prime}, g_{2}, g_{1}$ of the $S U(3)_{C}^{\prime} \otimes S U(2)_{W} \otimes U(1)_{Y}$. The coefficients of the oneloop beta functions $\beta_{a}=\left(b_{a} / 16 \pi^{2}\right) g_{a}^{3}$ are given by $\left(b_{1}, b_{2}, b_{3}\right)=(51 / 5,1,0)$. Note that the gauge coupling of $S U(3)_{C}^{\prime}$ is not asymptotically free but remains large for any energy scale. The gauge couplings for $S U(2)_{W}$ and $U(1)_{Y}$ both increase at higher scale and cross $g_{3}^{\prime}$ around the GUT scale [33].

This change in the running gauge couplings influences the supersymmetry breaking parameters obtained at the low energy scale significantly. Figure 4 shows the change of the supersymmetry breaking mass spectra obtained at low energy of the gaugino masses $M_{a}(a=1,2,3)$, the squark masses $m_{\tilde{Q}_{3}}, m_{\tilde{u}_{3}}$ and the right-handed selectron mass $m_{\tilde{e}}$ with respect to the messenger scale $\Lambda_{\text {mess }}$. As was mentioned just above, the gaugino mass $M_{3}$ is the same as $M_{3}^{\prime}$ through the SSB. It is seen that the selectron turns out to be much heavier than the bino in the high energy gauge mediation, while both are light in the low energy mediation. It will be found in the next section that the masses of the stop particles should be as large as $1 \mathrm{TeV}$ in order to satisfy the lightest Higgs mass bound. Then the

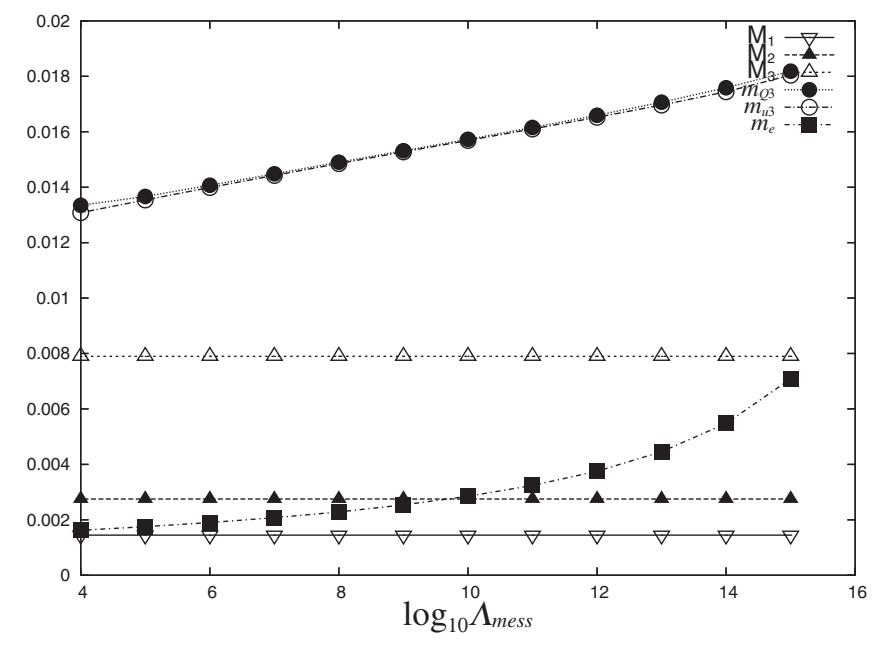

FIG. 4. The supersymmetry breaking masses $\left[M_{a}(a=1,2,3)\right.$ : the gaugino masses, $m_{\tilde{Q}_{3}}, m_{\tilde{u}_{3}}$ : the third generation squark masses, $m_{\tilde{e}}$ : the right-handed selectron mass] obtained at low energy for various messenger scales $\Lambda_{\text {mess }}$ are shown. Here we take $B_{S}=\left\langle F_{S}\right\rangle /\langle S\rangle=1$ as the unit.

bino mass is given above the experimental bound $M_{1}>$ $46 \mathrm{GeV}$.

\section{FINE-TUNING IN THE EXTRA $S U(3)$ MODEL}

\section{A. IR fixed point and running of $\boldsymbol{y}_{\Phi}$}

In this section, we evaluate the degree of fine-tuning required in the extra $S U(3)$ model explicitly, and see whether it is really improved as we expected in the previous section. The fine-tuning is determined by the size of $\left|m_{H_{u}}^{2}\right|$ at the weak scale. The induced value of $m_{H_{u}}^{2}$ by the gauge mediation is rather changeable with respect to the messenger scales, since the gauge couplings for $S U(2)_{W}$ and $U(1)_{Y}$ increase for the higher scale. However, a more important point is the renormalization of $m_{H_{u}}^{2}$ below the messenger scale. In practice, the renormalization is found to rely on the running behavior of the Yukawa coupling $y_{\Phi}$, although the scalar mass is not enhanced owing to the partial GMSB.

We mentioned that there is an IR attractive fixed point in the extra $S U(3)_{\text {ex }}$ gauge sector in the previous section. The couplings at the fixed point may be given as $\alpha_{\mathrm{ex}}=\alpha_{\mathrm{ex}}^{*}=$ $27 \pi / 64$ and $\alpha_{y_{\Phi}}=\alpha_{y_{\Phi}}^{*}=3 \pi / 8\left(\alpha_{\mathrm{ex}}=g_{\mathrm{ex}}^{2} / 4 \pi, \alpha_{y_{\Phi}}=\right.$ $\left.\left|y_{\Phi}\right|^{2} / 4 \pi\right)$, if we use the two-loop RG equations [19]. We find that the values of the fixed point couplings are fairly large, though the perturbative approximation is not reliable there. Even if these couplings are asymptotically free, they grow up towards IR and approaches the fixed point values.

In the followings, we consider two typical cases;

(1) Both the couplings stay on the fixed point, $\alpha_{\mathrm{ex}}=$ $\alpha_{\mathrm{ex}}^{*}$ and $\alpha_{y_{\Phi}}=\alpha_{y_{\Phi}}^{*}$, at all the scale below $\Lambda_{\text {mess }}$.

(2) Both the couplings $\alpha_{\mathrm{ex}}$ and $\alpha_{y_{\Phi}}$ are given as very small at $\Lambda_{\text {mess }}$ and rapidly increase near $\mathrm{TeV}$ scale. 
TATSUO KOBAYASHI et al.

We take the decoupling mass scale to be $5 \mathrm{TeV}$ in the analysis. The supersymmetry breaking parameters at the weak scale are evaluated by using the RG equations of the MSSM with the induced top Yukawa coupling $y_{t}^{\text {eff }}=1.0$ below $5 \mathrm{TeV}$.

\section{B. Case (1)}

The gauge and Yukawa couplings in the extra sector are set on their fixed point values. It is noted that not only the gaugino mass $M_{\text {ex }}$ but also the $A$-parameter corresponding to the Yukawa coupling $y_{\Phi}, A_{\Phi}$, are vanishing [34]. If we neglect the gaugino masses $M_{1}$ and $M_{2}$, which give minute corrections indeed, the one-loop RG equations for the scalar masses $m_{\Phi}^{2}, m_{\phi}^{2}$ and $m_{H_{u}}^{2}$ are given by

$$
\frac{d}{d \ln \Lambda}\left(\begin{array}{c}
m_{\Phi}^{2} \\
m_{\phi}^{2} \\
m_{H_{u}}^{2}
\end{array}\right)=\frac{\left|y_{\Phi}^{2}\right|}{8 \pi^{2}}\left(\begin{array}{ccc}
1 & 1 & 1 \\
2 & 2 & 2 \\
3 & 3 & 3
\end{array}\right)\left(\begin{array}{c}
m_{\Phi}^{2} \\
m_{\phi}^{2} \\
m_{H_{u}}^{2}
\end{array}\right) .
$$

It is found that a general solution of this equation is given by a superposition of two independent constant modes and one mode decreasing very rapidly towards IR. Therefore the soft masses obtained at low energy are always of the same order of their initial values, which are also independent of the messenger scale.

Here we should mention validity of this one-loop analysis, since the fixed point couplings are not very small. It has been also shown in the all order of perturbation [35] that each beta function of the soft scalar mass is vanishing at low energy and the masses satisfy the sum rule,

$$
m_{\Phi}^{2}+m_{\phi}^{2}+m_{H_{u}}^{2} \rightarrow 0 .
$$

It is noted that the solutions of the above RG equations also respect these properties. Thus, it is certain that the soft masses at low energy are independent of the messenger scale. Indeed, the one-loop approximation is not totally reliable quantitatively. However, this aspect of the soft masses is expected to be seen by the one-loop RG equations.

Figure 5 shows the actual running behavior of the soft scalar masses, $m_{\Phi}^{2} /\left|m_{\Phi}\right|, m_{\phi}^{2} /\left|m_{\phi}\right|$ and $m_{H_{u}}^{2} /\left|m_{H_{u}}\right|$, obtained by solving the one-loop RG equations for the extra $S U$ (3) model with a high messenger scale. Their initial values are given according to the GMSB of this model and, therefore are fairly large. The scale of the supersymmetry breaking parameters are fixed so as to lead the lightest Higgs mass of the experimental bound, $114.4 \mathrm{GeV}$.

It is seen that $m_{H_{u}}^{2}$ becomes negative quickly just below the messenger scale and turns to be a constant. The size of the Higgs scalar mass obtained at the weak scale is not so small, since it receives another sizable negative contribution below the decoupling scale. For the models with a lower messenger scale, the soft mass $\left|m_{H_{u}}^{2}\right|$ stabilizes at a much smaller value. Nevertheless, the lightest Higgs mass bound is more restrictive due to the small $A$-parameter and
PHYSICAL REVIEW D 74, 015002 (2006)

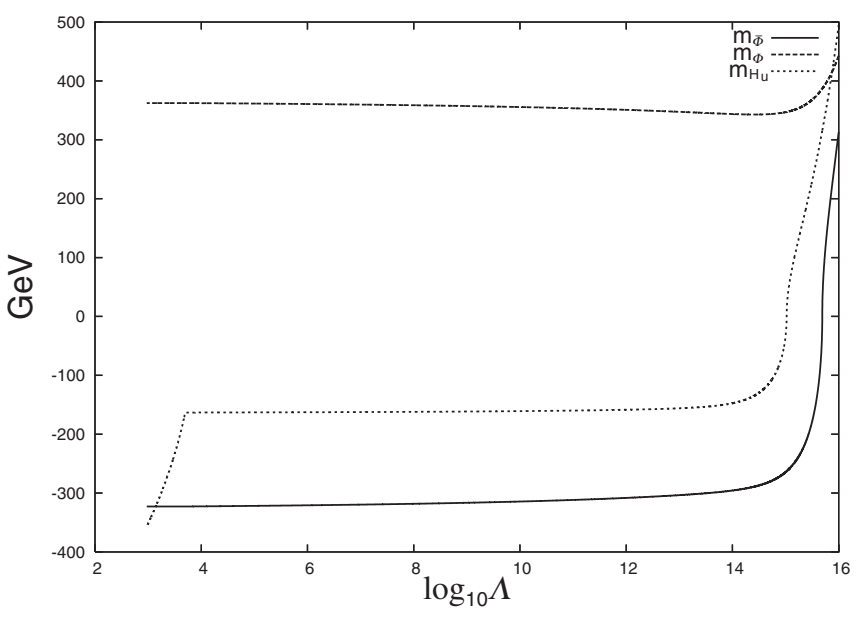

FIG. 5. The RG flows of the soft scalar masses, $m_{\Phi}^{2} /\left|m_{\Phi}\right|$, $m_{\phi}^{2} /\left|m_{\phi}\right|$ and $m_{H_{u}}^{2} /\left|m_{H_{u}}\right|$, obtained by solving the one-loop RG equations for the extra $S U(3)$ model are shown in the case of a high messenger scale.

makes the fine-tuning worse. As a result the fine-tuning of about $3 \%$ is found to be still required even at the best. Thus we may say that the models of Case (1) do not help to ameliorate the fine-tuning problem as expected naively.

\section{Case (2)}

Next we consider the case that both the couplings $\alpha_{\mathrm{ex}}$ and $\alpha_{y_{\Phi}}$ increase near the TeV scale. To be explicit, we examine the case with $\alpha_{\mathrm{ex}} \sim \alpha_{\mathrm{ex}}^{*}$ and $\alpha_{y_{\Phi}}=1 / \pi\left(y_{\Phi}=2\right)$ at the decoupling scale as an example. The RG flows of these couplings $g_{\text {ex }}$ and $y_{\Phi}$ are shown in Fig. 6. In general, it is not necessary for the extra gauge coupling $\alpha_{\text {ex }}$ also increases near the decoupling scale. The gauge coupling can approach the fixed point value at some higher energy

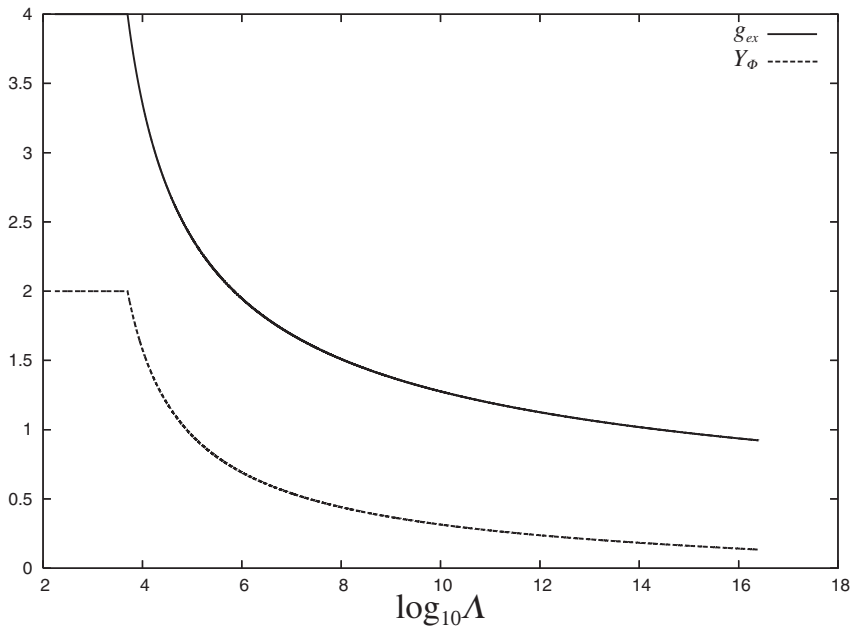

FIG. 6. The RG flows of the gauge couplings $g_{\text {ex }}$ and the Yukawa coupling $y_{\Phi}$ in the extra sector are shown in the case of $\alpha_{\mathrm{ex}} \sim \alpha_{\mathrm{ex}}^{*}$ and $\alpha_{y_{\Phi}}=1 / \pi\left(y_{\Phi}=2\right)$ at the decoupling scale $5 \mathrm{TeV}$. 
scale. The required feature is that the coupling $y_{\Phi}$ increases near the decoupling scale. This may be realized with a small initial coupling properly chosen at the GUT scale without recourse to fine-tuning.

Since the Yukawa coupling $y_{\Phi}$ becomes sizable only at the fairly low energy scale, $10^{3-4} \mathrm{GeV}$, the Higgs field is almost completely decoupled from the extra sector. Therefore these soft scalar masses are driven only by the gaugino contributions of the $S U(2)_{W}$ and the $U(1)_{Y}$, which are rather small even at high energy scale. The RG flows of the soft scalar masses $m_{\Phi}, m_{\phi}, m_{H_{u}}^{2} /\left|m_{H_{u}}\right|$ are shown in Fig. 7. The scale of the parameters are fixed so as to satisfy the lightest Higgs mass bound again. It is seen that the soft mass of $m_{H_{u}}^{2}$ is almost constant above the decoupling scale and receive the negative corrections through the Yukawa interactions of $y_{\Phi}$. Consequently, the low energy Higgs mass is found to be small and can be comparable with the weak scale.

As seen before, the lightest Higgs mass bound is rather sensitive to the parameter $A_{t}$. Note that the $A$-parameter $A_{\Phi}$ turns out to be $A_{t}$ effectively after the SSB. In practice, $A_{x}$, $A_{\bar{x}}$, and $B$-parameters for $\Phi$ and $\phi$ fields contribute to $A_{t}$ as well. Therefore we may obtain a large $A_{t}$, if the $B$-parameters are large. This may be a good point of this scenario, since large $A$-parameters are not induced in the GMSM. However we give an explicit evaluation of the fine-tuning only with $A_{\Phi}$ contribution, since the values of these extra parameters are uncertain anyway. The parameter $A_{\Phi}$ is generated below the messenger scale with following the RG equation,

$$
\frac{d A_{\Phi}}{d \ln \Lambda}=\frac{3\left|y_{\Phi}\right|^{2}}{4 \pi^{2}} A_{\Phi}+\frac{3 \alpha_{2}}{2 \pi} M_{2}+\frac{13 \alpha_{1}}{30 \pi} M_{1} .
$$

In Fig. 7, the RG flow of $A_{\Phi}$ is also shown. Below $5 \mathrm{TeV}$ the flow line is connected with the flow of $A_{t}$ in the MSSM.

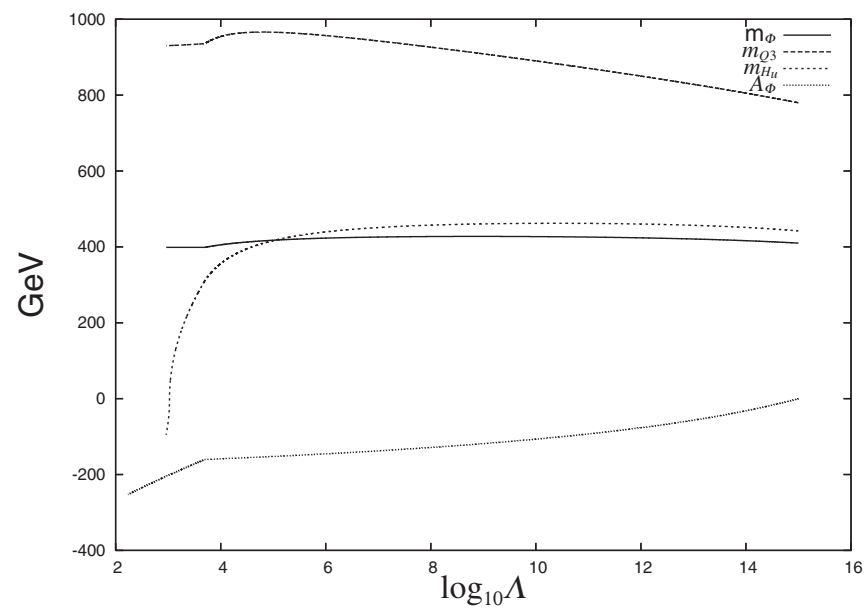

FIG. 7. The RG flows of the soft scalar masses $m_{\Phi}, m_{\phi}$, $m_{H_{u}}^{2} /\left|m_{H_{u}}\right|$ realized in the Case (2) are shown. The figure shows also the RG flow of $A_{\Phi}$ which is connected with the flow of $A_{t}$ of the MSSM at the decoupling scale.
It is seen that $A_{\Phi}$, or $A_{t}$, obtained at the weak scale is rather small even for the high energy GMSB. Nevertheless the lightest Higgs mass bound can be satisfied, since the stop particles are so heavy.

Now it is almost obvious that the fine-tuning problem is removed in this model, since the $\mu$-parameter required in order to realize the EWSB is also the weak scale. Figure 8 and 9 present the minimum degree of fine-tuning $\Delta_{\mu^{2}}$ required in the extra $S U(3)$ model for $\tan \beta=10$ and 5, respectively. (The data points with enough small degrees are not shown, since the model is safe from the fine-tuning anyway.) In the both figures, the upper lines show the minimum degrees to satisfy the lightest Higgs mass bound and the lower lines show the minimum degrees to satisfy the selectron mass bound.

These results tell us the following. The lightest Higgs mass bound always imposes the dominant constraint. However, the extra $S U(3)$ models with a large value of $\tan \beta$ is almost free from the severe fine-tuning for any messenger scale. It is also shown that $\mu$-parameter becomes smaller as the messenger scale goes higher, and is comparable with the weak scale for the high messenger scale GMSB.

Even in the case of $\tan \beta=5$, the degree of fine-tuning is reduced significantly compared with the ordinary MSSM. However, it is found that the minimum degree of finetuning increases rapidly for $\tan \beta<5$. This is because the required stop mass is so heavy (e.g. $2.5 \mathrm{TeV}$ for $\tan \beta=3$ ) as to induce a huge correction to the Higgs soft mass below the decoupling scale. The cancellation of the SM correction becomes also significant with such a large supersymmetry breaking scale anyway. Thus we may conclude that the natural models are obtained for a $\tan \beta$ more than 5 or so.

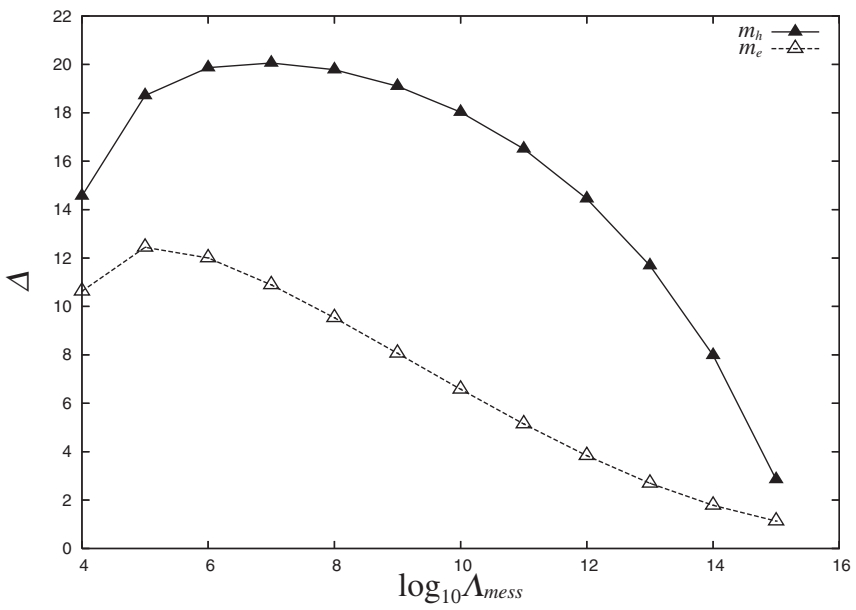

FIG. 8. The minimum degrees of fine-tuning required in the extra $S U(3)$ models with various messenger scales are shown in the case of $\tan \beta=10$. The conditions for the lines are the same as in Fig. 1. The data points for $\Lambda_{\text {mess }}=10^{16} \mathrm{GeV}$ are not given, since the EWSB solution is not realized. 
TATSUO KOBAYASHI et al.

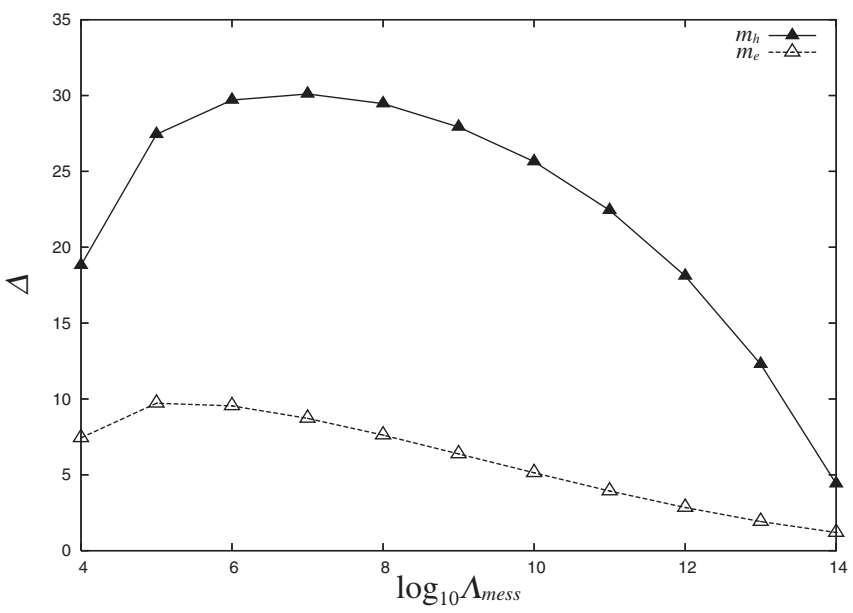

FIG. 9. The minimum degrees of fine-tuning in the case of $\tan \beta=5$ are shown in the same way as Fig. 8 .

\section{EXTENSION TO THE NEXT-TO-MINIMAL MODEL}

\section{A. Fine-tuning problem in the NMSSM}

The $\mu$-parameter as well as the $B$-parameter of the Higgs sector must be the same order of the soft supersymmetry breaking parameters in order to realize the EWSB. Therefore it would be natural to think that the $\mu$-parameter also has some origin related with the supersymmetry breaking. In the GMSB models, this problem appears to be a harder puzzle, since the generated $B$-parameter is given naively by $B_{S}=\left\langle F_{S}\right\rangle /\langle S\rangle$, which is too large for the Higgs potential. The simplest mechanism to solve this problem would be the NMSSM [12], while there have been proposed some other mechanisms [11].

The NMSSM introduces a SM-gauge singlet field $N$ replacing the $\mu$ term with the Yukawa term in the superpotential given by (5). Then the $\mu$-parameter may be generated through a nonvanishing vev of $N$ as $\mu=$ $\lambda_{H}\langle N\rangle$. However this vev breaks the global $U(1)_{P Q}$ symmetry of the superpotential spontaneously and, therefore, gives rise to the EW-scale axion, which is excluded experimentally [36]. Here we avoid this problem by breaking the $U(1)_{P Q}$ symmetry explicitly with introducing an additional term,

$$
\delta W=\frac{k}{3} N^{3},
$$

where the parameter $k$ is supposed to be comparable with $\lambda_{H}$.

We should mention here the new possibility for solving the fine-tuning problem proposed recently in Ref. [37]. When the pseudo-NG boson $a$ is very light, decay of the SM-like light Higgs boson $h$ to $a, h \rightarrow a a$, can be dominant over decay to the bottom quarks, $h \rightarrow b \bar{b}$. Then the Higgs may be missed and the mass can be lower than the bound of $114 \mathrm{GeV}$. However this possibility opens only
PHYSICAL REVIEW D 74, 015002 (2006)

when the coupling $k$ is rather small to make the pseudo-NG boson of the broken $U(1)_{\mathrm{PQ}}$ sufficiently light. Then a large $A$-parameter is required to raise the singlino and Higgsinos above LEP bounds [30], which is not realized in the GMSB. Therefore we do not consider the cases with a small $k$ in our discussion.

So far the fine-tuning required in the NMSSM has been investigated minutely in Refs. $[9,10]$. Here let us review the essence of their argument and give an analytic expression for the fine-tuning degrees by using somewhat rough approximations.

The parameters which we can tune freely are the Yukawa couplings $\lambda_{H}$ and $k$ only in the GMSB. Note that $\mu$ or $\tan \beta=v_{u} / v_{d}$ is not a free parameter any more, but is determined dynamically by the scalar potential. In order to discuss the fine-tuning, we must express the solution for $v^{2}$ in terms of the Yukawa couplings first. Then we examine stability of the solution under a small variation of the couplings.

The potential of the NMSSM model is written down as

$$
\begin{aligned}
V= & \left|\lambda_{H} H_{d} H_{u}-k N^{2}\right|^{2}+\lambda_{H}^{2}|N|^{2}\left(\left|H_{d}\right|^{2}+\left|H_{u}\right|^{2}\right) \\
& +\frac{g^{2}}{8}\left(H_{d}^{\dagger} \tau^{a} H_{d}+H_{u}^{\dagger} \tau^{a} H_{u}\right)^{2}+\frac{g^{\prime 2}}{8}\left(\left|H_{d}\right|^{2}-\left|H_{u}\right|^{2}\right)^{2} \\
& +m_{H_{u}}^{2}\left|H_{u}\right|^{2}+m_{H_{d}}^{2}\left|H_{d}\right|^{2}+m_{N}^{2}|N|^{2} \\
& -\left(\lambda_{H} A_{\lambda_{H}} N H_{u} H_{d}+\text { h.c. }\right)-\left(\frac{k}{3} A_{k} N^{3}+\text { h.c. }\right)+\cdots,
\end{aligned}
$$

where the ellipsis denotes higher order terms. Then the minimization conditions for the neutral scalar components are found to be

$$
\begin{aligned}
& \lambda_{H}^{2} x^{2}=-\frac{g^{2}+g^{\prime 2}}{8} v^{2}+\frac{m_{H_{d}}^{2}-m_{H_{u}}^{2} \tan ^{2} \beta}{\tan ^{2} \beta-1} \\
& A_{\lambda_{H}} \lambda_{H} x=\left(m_{H_{d}}^{2}+m_{H_{u}}^{2}+2 \lambda_{H}^{2} x^{2}\right) \frac{\sin 2 \beta}{2} \\
& 2 k^{2} x^{2}=\lambda_{H} v^{2}\left(k \sin 2 \beta-\lambda_{H}\right)-m_{N}^{2}+A_{\lambda_{H}} \lambda_{H} v^{2} \frac{\sin 2 \beta}{2 x} \\
& +k A_{k} x \text {, }
\end{aligned}
$$

where $v_{u}=\left\langle H_{u}\right\rangle, v_{d}=\left\langle H_{d}\right\rangle,\left(v^{2}=v_{u}^{2}+v_{d}^{2}\right)$ and $x=$ $\langle N\rangle$. It is seen that the first and the second equations are identical with the conditions in the MSSM by replacing as $\lambda_{H} x=\mu$ and $A_{\lambda_{H}}=B$.

Here we shall approximate the solutions of the above equations by performing some simplifications. First we neglect the $A$-parameters, since $A_{\lambda_{H}}$ is much smaller than $v$ and $A_{k}$ is negligible in the GMSB irrespective of the messenger scale. Next we simplify the conditions further by setting as $m_{H_{u}}^{2}+m_{H_{d}}^{2} \sim m_{H_{u}}^{2}$ and $\sin 2 \beta \sim 2 / \tan \beta$. Although these simplifications are not always good quan- 
titatively, we may find roughly how strong fine-tuning is required. Then the equations are reduced to be

$$
\begin{gathered}
\mu^{2}+\frac{c}{2} v^{2}+m_{H_{u}}^{2}=0, \\
\kappa \tan \beta \mu^{2}+\left(c-\lambda_{H}^{2}\right) v^{2}+m_{H_{u}}^{2}=0, \\
2 \kappa^{2} \mu^{2}+\lambda_{H}^{2} v^{2}\left(1-\frac{2 \kappa}{\tan \beta}\right)+m_{N}^{2}=0,
\end{gathered}
$$

where $\mu^{2}=\lambda_{H}^{2} x^{2}, \quad c=\left(g^{2}+g^{2}\right) / 4$, and $\kappa=k / \lambda_{H}$. After eliminating $\mu^{2}$ from these equations, the conditions are reduced further to

$$
\begin{gathered}
F_{1} \equiv-(1-\kappa \tan \beta) m_{H_{u}}^{2}-\left(1-\frac{\kappa}{2} \tan \beta\right) \alpha v^{2}+\lambda_{H}^{2} v^{2}=0 \\
F_{2} \equiv 2 \kappa^{2}\left(-m_{H_{u}}^{2}-\frac{\alpha}{2} v^{2}\right)+\lambda_{H}^{2} v^{2}\left(1-\frac{2 \kappa}{\tan \beta}\right)+m_{N}^{2}=0 .
\end{gathered}
$$

As seen before, the soft scalar mass $\left|m_{H_{u}}\right|$ is far larger than the weak scale in the GMSB. Therefore we first consider the conditions so that the Yukawa couplings $\lambda_{H}$ and $k$ should satisfy in order to have $v^{2} \ll\left|m_{H_{u}}^{2}\right|$ as a solution. However, it has been known that the phenomenologically viable spectrum cannot be obtained in the GMSB $[9,10]$. The reason is that the Yukawa interaction with $\lambda_{H}$ itself cannot drive the soft mass for $N, m_{N}^{2}$, to be sufficiently negative at the weak scale.

From the Eq. (40), it is found that $\mu^{2} \sim\left|m_{H_{u}}^{2}\right| \gg v^{2}$. Meanwhile the Eq. (42) may be rewritten as [9]

$$
\begin{aligned}
-2 \kappa^{2} \mu^{2}= & \lambda_{H}^{2} v^{2}\left(1-\frac{2 \kappa}{\tan \beta}\right)+m_{N}^{2} \\
\simeq & \lambda_{H}^{2}\left[v^{2}\left(1-\frac{2 \kappa}{\tan \beta}\right)-\frac{1}{4 \pi^{2}}\left(m_{H_{u}}^{2}\left(\Lambda_{\text {mess }}\right)\right.\right. \\
& \left.\left.+m_{H_{d}}^{2}\left(\Lambda_{\text {mess }}\right)\right) \log \frac{\Lambda_{\text {mess }}}{m_{H_{d}}}\right]
\end{aligned}
$$

where we evaluated $m_{N}^{2}$ by using the one-loop perturbation. However this equation cannot be satisfied even with a very large messenger scale $\Lambda_{\text {mess }}$, since $\kappa$ cannot be taken so small. Therefore the NMSSM is often modified to have also a pair of extra vectorlike color-triplet fields coupled with the singlet $N[8,11]$. Then the low energy scalar potential can lead to a viable EWSB with $m_{N}^{2}$ sufficiently negative. In Refs. $[9,10]$, the fine-tuning problem has been investigated in the NMSSM model with the extra "quarks" and was found to be more serious than in the MSSM.

Hence we may consider the cases with $\left|m_{N}^{2}\right| \sim\left|m_{H_{u}}^{2}\right| \gg$ $v^{2}$. Then it is seen from Eqs. (43) and (44) that such a hierarchical difference among the mass scales can appear, only when the ratio of the couplings $\lambda_{H}$ and $k$ happens to be

$$
\kappa^{2}=\frac{k^{2}}{\lambda_{H}^{2}} \sim \frac{\left|m_{N}^{2}\right|}{2\left|m_{H_{u}}^{2}\right|} .
$$

If the parameter $\kappa$ deviates from this relation, then the solution of $v^{2}$ becomes comparable with $\left|m_{H_{u}}^{2}\right|$ immediately. This is the origin of the strong fine-tuning. We also note that $\tan \beta$ of the solution is also fixed to be

$$
\tan \beta \sim 1 / \kappa
$$

approximately. This is the reason why $\tan \beta$ cannot be large in the NMSSM models in general.

The response of the solutions $\left(v^{2}, \tan \beta\right)$ under a slight shift of e.g. $\kappa$ satisfies the following relations,

$$
\begin{gathered}
\kappa \frac{\partial F_{1}}{\partial \kappa}+\kappa \frac{\partial v^{2}}{\partial \kappa} \frac{\partial F_{1}}{\partial v^{2}}+\kappa \frac{\partial \tan \beta}{\partial \kappa} \frac{\partial F_{1}}{\partial \tan \beta}=0, \\
\kappa \frac{\partial F_{2}}{\partial \kappa}+\kappa \frac{\partial v^{2}}{\partial \kappa} \frac{\partial F_{2}}{\partial v^{2}}+\kappa \frac{\partial \tan \beta}{\partial \kappa} \frac{\partial F_{2}}{\partial \tan \beta}=0 .
\end{gathered}
$$

By solving these equations, we may evaluate the degree of fine-tuning in order to realize the specific solution of $v^{2}$. Since we consider the solutions with $v^{2} \ll\left|m_{N}^{2}\right| \sim\left|m_{H_{u}}^{2}\right|$, the degree is found to be approximated as

$$
\Delta_{\kappa}\left(v^{2}\right)=\left|\frac{\kappa}{v^{2}} \frac{\partial v^{2}}{\partial \kappa}\right| \sim\left|\frac{2 \kappa^{2}}{\kappa^{2}-\lambda_{H}^{2}\left(1-2 \kappa^{2}\right) / c}\right| \frac{2\left|m_{H_{u}}^{2}\right|}{M_{Z}^{2}} .
$$

Although we should examine also the deviation of $\lambda_{H}$, the solutions are found to be rather stable. Here we note that the quantity $2\left|m_{H_{u}}^{2}\right| / M_{Z}^{2}$ is nothing but the degree of finetuning for the MSSM case. This is the analytic expression for the fine-tuning index for the NMSSM in the GMSB. We may certify the validity of this formula by solving the coupled equations numerically, (37)-(39), with incorporating all of the parameters given by the GMSB framework. It is found to be good as long as $\left|m_{H_{u}}^{2}\right|,\left|m_{N}^{2}\right| \gg v^{2}$.

The Yukawa coupling $\lambda_{H}$ cannot be taken to be more than 0.5 , otherwise the running coupling hits the Landau pole well before the GUT scale. Therefore, unless the denominator of the index given by (50) happens to be small, the fine-tuning in the NMSSM is the same as that obtained in the MSSM with the same $\tan \beta$ up to the factor of $O(1)$ [10]. Consequently, the fine-tuning required in the NMSSM models is found to be no better than in the MSSM.

The crucial difference between the MSSM and the NMSSM is the value of $\tan \beta$. However, the term of $\lambda_{H} N H_{u} H_{d}$ in the superpotential lifts up the lightest Higgs mass bound for the small $\tan \beta$ case as

$$
m_{h^{0}}^{2} \geq\left(M_{Z}^{2} \cos ^{2} 2 \beta+\lambda_{H}^{2} v^{2} \sin ^{2} 2 \beta\right)+(\text { loop}) .
$$

Therefore the constraint on the stop masses from the Higgs 
mass bound becomes almost independent of $\tan \beta$ for $\lambda_{H} \sim$ 0.5 . Unless the coupling $\lambda_{H}$ is given to be so large, the finetuning is very severe in the NMSSM models [14].

\section{B. Extension of the extra $S U(3)$ model}

Lastly we consider the fine-tuning in the extra $S U(3)$ model modified so that the supersymmetric mass parameters are generated through vev of a singlet $N$. The superpotential is given explicitly by

$$
\begin{aligned}
W= & y_{t} Q \bar{u} H_{u}+\lambda_{H} N H_{d} H_{u}-\frac{1}{3} k N^{3}+y_{\Phi} \Phi \bar{\phi} H_{u} \\
& +\lambda_{\Phi} N \Phi \bar{\Phi}+\lambda_{\phi} N \phi \bar{\phi}+\lambda_{\Omega} N \Omega \bar{\Omega} .
\end{aligned}
$$

The decoupling masses of the extra vectorlike fields as well as the $\mu$ parameter are induced through the singlet vev as $M_{\Phi}=\lambda_{\phi}\langle N\rangle$, etc. Here, however, these masses should be much larger than $\mu$, e.g. a few $\mathrm{TeV}$. It is interesting that such a hierarchy may be also generated by the strong gauge dynamics in the extra sector. The Yukawa couplings, $\lambda_{\Phi}$, $\lambda_{\Phi}$, and $\lambda_{\Omega}$, are also enhanced by the strong gauge coupling $\alpha_{\mathrm{ex}}$ similarly to $y_{\Phi}$, while $\lambda_{H}$ is not enhanced. Therefore a large discrepancy among these couplings arises at the low energy scale naturally.

It may be an advantage for the extra $S U(3)$ model to contain the extra vectorlike fields coupled with $N$ from the beginning. Owing to these couplings, the soft scalar mass $m_{N}^{2}$ can be driven to be sufficiently negative at the low energy scale. In practice, the viable models for the EWSB are found to be obtained for appropriate choices of the Yukawa couplings.

The main difference between the present model and the conventional NMSSM model examined in Refs. [9,10] is that the size of the soft scalar mass $\left|m_{H_{u}}^{2}\right|$ obtained at the weak scale is not so enhanced by the large stop masses. That is because the extended model also enjoys the nature that the radiative correction to $m_{H_{u}}^{2}$ can be effectively cutoff around the decoupling scale. On the other hand, however, the problem is that the coupling $\lambda_{H}$ cannot be taken as large as 0.5. First the Yukawa couplings $\lambda_{\Phi}$ and $\lambda_{\phi}$ should be less than 0.5 , otherwise these Yukawa interactions induce a sizable soft mass $\left|m_{N}\right|$, and, therefore, the solution of $\tan \beta$ becomes too small. Then the coupling $\lambda_{H}$ should be smaller than about 0.05 to make the mass ratio $\lambda_{\Phi} / \lambda_{H}=M_{\Phi} / \mu$ sufficiently large, otherwise the decoupling scale of the extra sector is too low to pass the EW precision tests.

Thus, unfortunately the above extension of the extra $S U$ (3) model to the NMSSM type appears to be unsuccessful. The fine-tuning is so severe as in the MSSM with a small $\tan \beta$ as seen in the previous section. However, this situation can be improved remarkably, if the Yukawa coupling $\lambda_{H}$ is allowed to be somewhat large as $0.3-0.5$. Then some other mechanisms to generate the decoupling scale of a few $\mathrm{TeV}$ are required. We leave this problem in the NMSSM model to the future studies.

\section{CONCLUSION}

In this paper we first studied the degree of fine-tuning in the GMSB with an arbitrary messenger scale. Specifically we took the lightest Higgs mass bound by LEP into consideration by using the 2-loop approximation. The spectra of all soft supersymmetry breaking parameters obtained at the weak scale are completely fixed up to their overall scale $B_{S}$. Therefore we examined the fine-tuning with respect to the $\mu$-parameter required so as to realize the EWSB.

Then it was found that the constraint on the soft masses by the Higgs mass bound is far more restrictive than by the selectron mass bound for the entire range of the messenger scale, and the fine-tuning stronger than a few percent is required at the very best. The degree becomes bigger as the messenger scale is lowered, because the $A$-parameter $A_{t}$ is given to be small. Moreover, the model with a small value of $\tan \beta(\leq 5)$ needs an extreme fine-tuning of the $\mu$-parameter.

In order to solve this problem, we studied a new scenario in which the top Yukawa coupling is induced at $\mathrm{TeV}$ scale through mass mixing with some extra matter fields coupled with the Higgs field. We also applied the partial GMSB mechanism so that the extra gauge sector is sequestered from the supersymmetry breaking sector. In the explicit model of the Case (2) presented in Sec. IV, the radiative correction to the Higgs soft mass can be cutoff effectively at the TeV scale. Then the Higgs soft mass is found to be comparable with the weak scale and, therefore, renders the fine-tuning of the $\mu$-parameter unnecessary, even if the stop masses are quite large. In practice, we found that the natural models are obtained for $\tan \beta$ more than 5 or so.

We also examined the oblique corrections induced by the mixing with the extra fields and checked that the models are safe as long as the extra particles are heavier than $1 \mathrm{TeV}$. The feature of this model is that the Higgs scalars and the Higgsinos are as light as the weak scale, while the squarks are quite heavy. Moreover, the sleptons can be heavier than the bino for a high messenger scale. This is because the running behaviors of the SM gauge couplings are modified by the presence of the extra fields.

The extension to the NMSSM is supposed to be the easiest way to avoid the $\mu$-problem in the GMSB framework. It has been known, however, that the additional correction by the extra quarks is necessary to make a viable model. In the extra $S U(3)$ model, the fields in the extra gauge sector coupled with the singlet field play this role and the realistic EWSB can take place.

Unfortunately, the extended model turns out to suffer fine-tuning, because $\tan \beta$ determined by the EWSB vacuum is small. In the explicit model the Yukawa coupling of the singlet with the Higgs field $\lambda_{H}$ is taken to be rather small in order to make the decoupling mass heavy. 
However, if $\lambda_{H}$ can be enhanced, the fine-tuning problem may be resolved just as in the MSSM with large $\tan \beta$. The improvement of the NMSSM model in this way is left for the future study.

\section{ACKNOWLEDGMENTS}

T. K. and H. T. are supported in part by the Grants-in-Aid for Scientific Research No. 17540251 and No. 40192653 respectively from the Ministry of Education, Science, Sports and Culture, Japan. T. K. is also supported in part by the Grant-in-Aid for the 21st Century COE "The Center for Diversity and Universality in Physics".

Note Added:-As this paper was prepared, we found that a comprehensive paper by R. Kitano and Y. Nomura [23] appeared and our study given in Sec. II overlaps in part.
[1] R. Barbieri and G.F. Giudice, Nucl. Phys. B306, 63 (1988).

[2] P. H. Chankowski, J. R. Ellis, and S. Pokorski, Phys. Lett. B 423, 327 (1998); Nucl. Phys. B544, 39 (1999); G. L. Kane and S.F. King, Phys. Lett. B 451, 113 (1999); M. Bastero-Gil, G. L. Kane, and S. F. King, Phys. Lett. B 474, 103 (2000).

[3] ALEPH, DELPHI, L3, and OPAL Collaborations, Phys. Lett. B 551, 146 (2003).

[4] Y. Okada, M. Yamaguchi, and T. Yanagida, Phys. Lett. B 262, 54 (1991); H. E. Haber and R. Hempfling, Phys. Rev. Lett. 66, 1815 (1991); J. R. Ellis, G. Ridolfi, and F. Zwirner, Phys. Lett. B 262, 477 (1991).

[5] M. Carena, M. Quirós, and C. E. M. Wagner, Nucl. Phys. B461, 407 (1996); M. Carena, H.E. Haber, S. Heinemeyer, W. Hollik, C.E.M. Wagner, and G. Weiglein, Nucl. Phys. B580, 29 (2000).

[6] K. Inoue, A. Kakuto, H. Komatsu, and S. Takeshita, Prog. Theor. Phys. 67, 1889 (1982); 68, 927 (1982); 70, 330(E) (1983); 71, 413 (1984); L. E. Ibanez and G. G. Ross, Phys. Lett. 110B, 215 (1982); L. Alvarez-Gaume, M. Claudson, and M. B. Wise, Nucl. Phys. B207, 96 (1982).

[7] See for a review, e.g., J. L. Feng, hep-ph/0405215; see also N. Arkani-Hamed, A. Delgado, and G. F. Giudice, Nucl. Phys. B741, 108 (2006), and references therein.

[8] See for a review, e.g., G. F. Giudice and R. Rattazzi, Phys. Rep. 322, 419 (1999), and references therein.

[9] K. Agashe and M. Graesser, Nucl. Phys. B507, 3 (1997).

[10] A. de Gouvêa, A. Friedland, and H. Murayama, Phys. Rev. D 57, 5676 (1998).

[11] M. Dine and A. Nelson, Phys. Rev. D 48, 1277 (1993); M. Dine, A. Nelson, and Y. Shirman, Phys. Rev. D 51, 1362 (1995); M. Dine, A. Nelson, Y. Nir, and Y. Shirman, Phys. Rev. D 53, 2658 (1996); G. Dvali, G. F. Giudice, and A. Pomarol, Nucl. Phys. B478, 31 (1996).

[12] P. Fayet, Nucl. Phys. B90, 104 (1975); H. P. Nilles, M. Srednicki, and D. Wyler, Phys. Lett. 120B, 346 (1983); J. M. Frere, D. R. T. Jones, and S. Raby, Nucl. Phys. B222, 11 (1983); J. P. Derendinger and C. A. Savoy, Nucl. Phys. B237, 307 (1984); J. R. Ellis, J. F. Gunion, H. E. Haber, L. Roszkowski, and F. Zwirner, Phys. Rev. D 39, 844 (1989).

[13] A. Brignole, J. A. Casas, J. R. Espinosa, and I. Navarro, Nucl. Phys. B666, 105 (2003); J. A. Casas, J. R. Espinosa, and I. Hidalgo, J. High Energy Phys. 01 (2004) 008.

[14] R. Harnik, G. D. Kribs, D. T. Larson, and H. Murayama, Phys. Rev. D 70, 015002 (2004); S. Chang, C. Kalic, and
R. Muhbubani, Phys. Rev. D 71, 015003 (2005); A. Birkedal, Z. Chacko, and Y. Nomura, Phys. Rev. D 71, 015006 (2005); A. Delgado and T. M.P. Tait, J. High Energy Phys. 07 (2005) 023.

[15] P. Batra, A. Delgado, D. E. Kaplan, and T. M. P. Tait, J. High Energy Phys. 02 (2004) 043; 06 (2004) 032.

[16] P. J. Fox, A.E. Nelson, and N. Weiner, J. High Energy Phys. 08 (2002) 035; Z. Chacko, P. J. Fox, and H. Murayama, Nucl. Phys. B706, 53 (2005).

[17] A. Birkedal, Z. Chacko, and M. K. Gaillard, J. High Energy Phys. 10 (2004) 036; P. H. Chankowski, A. Falkowski, S. Pokorski, and J. Wagner, Phys. Lett. B 598, 252 (2004); Z. Berezhiani, P. H. Chankowski, A. Falkowski, and S. Pokorski, Phys. Rev. Lett. 96, 031801 (2006); T. Roy and M. Schmaltz, J. High Energy Phys. 01 (2006) 149; C. Csáki, G. Marandella, Y. Shirman, and A. Strumia, Phys. Rev. D 73, 035006 (2006).

[18] T. Kobayashi and H. Terao, J. High Energy Phys. 07 (2004) 026.

[19] T. Kobayashi, H. Nakano, and H. Terao, Phys. Rev. D 71, 115009 (2005).

[20] Y. Nomura, D. Poland, and B. Tweedie, Nucl. Phys. B745, 29 (2006).

[21] K. Choi, K. S. Jeong, T. Kobayashi, and K.i. Okumura, Phys. Lett. B 633, 355 (2006); R. Kitano and Y. Nomura, Phys. Lett. B 631, 58 (2005).

[22] R. Dermisek and H. D. Kim, hep-ph/0601036.

[23] R. Kitano and Y. Nomura, Phys. Rev. D 73, 095004 (2006).

[24] A. Falkowskoi, S. Pokorski, and M. Schmaltz, hep-ph/ 0604066; S. Chang, L. Hall, and N. Weiner, hep-ph/ 0604076

[25] J. L. Feng, K. T. Matchev, and T. Moroi, Phys. Rev. Lett. 84, 2322 (2000); Phys. Rev. D 61, 075005 (2000); J. L. Feng, K. T. Matchev, and F. Wilczek, Phys. Lett. B 482, 388 (2000).

[26] G. F. Giudice and R. Rattazzi, Nucl. Phys. B511, 25 (1998).

[27] In the case of $\left\langle F_{S}\right\rangle \sim\langle S\rangle^{2}$, the formulas (8) and (9) are modified with a factor of $O(1)$ [8].

[28] It is noted that the analyses of the degree of fine-tuning required in the GMSB shown in Refs. $[9,10]$ put emphasis on the slepton mass bound.

[29] N. Seiberg, Nucl. Phys. B435, 129 (1995); K. Intrilligator and N. Seiberg, Nucl. Phys. B, Proc. Suppl. 45, 1 (1996).

[30] LEP Collaborations, ALEPH, DELPHI, L3, OPAL, and 
LEP Electroweak Working Group, hep-ex/0511027.

[31] B. A. Dobrescu and C. T. Hill, Phys. Rev. Lett. 81, 2634 (1998); R. S. Chivukula, B. A. Dobrescu, H. Georgi, and C. T. Hill, Phys. Rev. D 59, 075003 (1999); H. Collins, A. K. Grant, and H. Georgi, Phys. Rev. D 61, 055002 (2000).

[32] See for a review, e.g., M. Schmaltz and D. Tucker-Smith, Annu. Rev. Nucl. Part. Sci. 55, 229 (2005).

[33] Unfortunately the gauge coupling unification does not hold exactly, because the hypercharge assignment is not fit for the $S U(5)$ representations. Though it is interesting to construct viable models which can be embedded into a GUT theory, it is beyond our present scope.

[34] Though the A-parameter is not generated from the begin- ning in the GMSB, it has been known that the $A$-parameters decrease rapidly at lower scale in the general superconformal field theories.

[35] A.E. Nelson and M. J. Strassler, J. High Energy Phys. 09 (2000) 030; 07 (2002) 021; T. Kobayashi and H. Terao, Phys. Rev. D 64, 075003 (2001); T. Kobayashi, H. Nakano, and H. Terao, Phys. Rev. D 65, 015006 (2002); T. Kobayashi, H. Nakano, T. Noguchi, and H. Terao, Phys. Rev. D 66, 095011 (2002).

[36] See for a recent analysis, e.g., L. J. Hall and T. Watari, Phys. Rev. D 70, 115001 (2004).

[37] R. Dermisek and J. F. Gunion, Phys. Rev. Lett. 95, 041801 (2005); S. Chang, P. J. Fox, and N. Weiner, hep-ph/ 0511250 ; P. C. Schuster and N. Toro, hep-ph/0512189. 